\title{
Discrimination against diacylglycerol ethers in lipase-catalyzed ethanolysis of shark liver oil
}

Óscar Fernández, Luis Vázquez *, Guillermo Reglero, Carlos F. Torres

Departament of Production and Characterization of Novel Foods

Institute of Food Science Research (CIAL) (CSIC-UAM)

9 Nicolás Cabrera. UAM Cantoblanco Campus

28049 Madrid, Spain

${ }^{*}$ Corresponding author:

Telephone: +34 (91) 0017-919

Fax: +34 (91) 0017-905

E-mail: luis.vazquez@uam.es 


\begin{abstract}
Lipase-catalyzed ethanolysis of squalene free shark liver oil has been investigated. The mentioned shark liver oil was comprised mainly of diacylglycerol ether and triacylglycerols. In order to test discrimination against diacylglycerol ether, up to ten different lipases were compared. The ratio oil to ethanol and lipase stability were also evaluated. Surprisingly, lipase from Pseudomonas stutzeri was the fastest biocatalyst among all assayed although poor discrimination against diacyl glycerol ether was attained. In this study, the best results in terms of selectivity and stability were obtained with immobilized lipase from Candida antarctica (Novozym 435). Ethanolysis reaction in the presence of Novozym 435 produced total disappearance of triacylglycerol and a final reaction mixture comprised mainly of diacyl glycerol ethers and monoacylglycerol ethers.
\end{abstract}

\title{
Keywords
}

Alkylglycerol

Ethanolysis

Ether lipids

Lipase

Shark liver oil 


\section{Introduction}

Glycerolipids are molecules that contain a glycerol backbone linked to different alkyl chains by different bond types. Depending on the number of chains linked to the glycerol molecule, glycerolipids can be classified into three categories: triradyl-, diradyl- and monoradylglycerols. Figure 1 shows the structural differences of the main subclasses of triradyl-, diradyl- and monoradyl-glycerols.

Alkylglycerols (AKG), monoacylglycerol ethers (MAGE), diacylglycerol ethers (DAGE), alkylglycerophospholipids and their derivatives, commonly known as ether lipids, have been the subject of much attention due to their special health-promoting effects in humans (Magnusson \& Haraldsson, 2011). Ether lipids have been used in the therapy of cancer (Andreesen, 1988), since they are potent antineoplastic agents which inhibit growth, show antimetastatic activity and induce differentiation and apoptosis in cancer cells (Diomede, Colotta, Piovani, Re, Modest \& Salmona, 1993). Immune stimulators properties have also been attributed to dietary ingestion of these substances (Palmblad, Samuelsson \& Brohult, 1990). In addition, recent studies indicate the these compounds could improve the bioavailability of othe lipid molecules such as butyric or omega-3 fatty acids (Martín, Morán-Valero, Señoráns, Reglero \& Torres, ; Torres, Vázquez, Señoráns \& Reglero, 2009). Hence, extent and type of esterification can play a significant role in their biological activity.

Ether lipids are widely distributed in marine and terrestrial animals, but they are not or rarely found in plant sources. Non-polar glyceryl ether lipids of the 1-O-alkyl-2,3-diacylsn-glycerol type or DAGE are major constituents in the liver oils of various species of elasmobranch fish such as dogfish and shark (Haraldsson \& Kristinsson, 1998). However, due to the current need to avoid shark overfishing, alternative sources of ether lipids have been identified. Hence, high contents of these compounds can be found in some species of pteropoda (Clione limacina) (Böer, Gannefors, Kattner, Graeve, Hop \& Falk-Petersen, 2005), liver of deep-sea squids (Berryteuthis magister) (Hayashi \& Kishimura, 2002), marine 
invertebrates (sponge Niphates digitalis) (Meimetis et al., 2011) and species of coral (Gersemia rubiformis) (Imbs, Demina \& Demidkova, 2006).

Ether lipids are commonly found in shark liver oil together with high amounts of squalene, TAG, and $\omega-3$ fatty acids. Since these compounds may provide similar bioactive effects in the human body, methods for purification of ether lipids from natural sources, to identify with accuracy their specific physiological role, are highly demanded. Isolation and purification of ether lipids from natural sources has shown to be difficult, mainly because of the presence of TAG, which have analogous structure, molecular weight, polarity and volatility.

Some common procedures used at large-scale to concentrate specific lipids from different raw materials are urea complexation (Senanayake \& Shahidi, 2000), low temperature crystallization (winterization) (Vázquez \& Akoh, 2011), supercritical fluid extraction (SFE) (Vázquez, Fornari, Señoráns, Reglero \& Torres, 2008) or short-path distillation (Vázquez \& Akoh, 2010). These methods are commonly based on differences in the molecular weight, degree of unsaturation, volatility and solubility in $\mathrm{CO}_{2}$ of the compounds. In these terms, ether lipids and TAG are similar molecules and its separation by using the mentioned technologies can be unfeasible since these compounds would have similar behavior.

Recently, our research group has been able to concentrate AKG by a two-step methodology based on the transesterification by ethanolysis of shark liver oil and subsequent SFE to purify the products (Vázquez et al., 2008). The transesterification reaction converts triacylglycerols and DAGE into the corresponding fatty acid ethyl esters (FAEE), plus AKG together with minor amounts MAGE and lower glycerides. Saponification has also been used to modify the original shark liver oil (Torres, Vázquez, Señoráns \& Reglero, 2007). As stated previously, saponification or ethanolysis reactions are necessary because of the presence of TAG that could interfere in the separation. However, no 
discrimination between DAGE and TAG were observed during these chemical transformations. Besides, significant amounts of unidentified material that may come in part, from undesired oxidation and/or polymerization reactions, were generated in these processes (Tenllado, Reglero \& Torres).

In order to modify only the TAG present in the shark liver oil avoiding the generation of undesired byproducts, the enzymatic technology has shown very interesting capabilities. Several advantages have been attributed to lipase-catalyzed reactions such as milder reaction conditions, non-toxic reactants and catalysts, and high specificity (Irimescu, Furihata, Hata, Iwasaki \& Yamane, 2001). The mildness that they offer in terms of temperature, $\mathrm{pH}$ and pressure most certainly protects them from partial destruction of their natural all-cis framework by oxidation, cis-trans isomerization or double-bond migrations (Halldorsson, Kristinsson \& Haraldsson, 2004). Numerous studies have demonstrated the efficiency of several lipases to effectively discriminate between lipids with slight differences in their structure (Shimada, Sugihara \& Tominaga, 2001). These processes are based on the specificity of different lipases that discriminate or act very weakly on the desired molecule. The different sources of lipases bring about different substrate specificities which are determined by the differences in their tridimensional structures, conformation, and active site flexibility making it possible to find versatile lipases for different purposes (Song, Qi, Hao \& Qu, 2008). Specificity of lipases is controlled by the molecular properties of the enzyme, structure of the substrate and factors affecting binding of the enzyme to the substrate (Jensen, DeJong \& Clark, 1983).

There are numerous reports in the literature describing the application of commercially available lipases to concentrate certain fatty acids or groups of fatty acids from marine oils in various types of hydrolysis, esterification and transesterification reactions (Halldorsson et al., 2004). Haraldsson et al. (Breivik, Haraldsson \& Kristinsson, 1997; Haraldsson, Kristinsson, Sigurdardottir, Gudmundsson \& Breivik, 1997) reported excellent 
results for concentration of PUFA by transesterification of fish oil with ethanol. In these studies, lipases from Pseudomonas showed the highest discrimination against EPA and DHA in fish oil with a stoichiometric amount of ethanol. Irimescu et al. (Irimescu et al., 2001; Irimescu, Iwasaki \& Hou, 2002) investigated ethanolysis reactions with Candida antarctica (Novozym 435) as catalyst to produce 2-monoacylglycerols from various polyunsaturated TAG. This study suggested that Novozym 435 showed strict positional regiospecificity in an excess amount of ethanol. Watanabe et al. (Watanabe, Shimada, Sugihara \& Tominaga, 1999) also used Candida antarctica in ethanolysis processes of tuna oil at $40{ }^{\circ} \mathrm{C}$. In their work the ethanol was added to the reaction mixture in three steps in order to avoid the inactivation of the catalyst. Yamane et al. (Piyatheerawong, Iwasaki, Xu \& Yamane, 2004) reported that an important factor that greatly affects the activity of biocatalysts in ethanolysis reactions is the amount of water in the reaction system. In this way, Xu et al. (Xu, Nordblad, Nielsen, Brask \& Woodley, 2011) described the negative effect caused by the accumulation of glycerol in ethanolysis of rapeseed oil. So it was confirmed that glycerol can inhibit immobilized lipases, most likely by clogging of the catalyst particles.

In the present study, lipase-catalyzed transesterification processes by ethanolysis of shark liver oil (squalene-free) was investigated. The main aim of this work was to promote and study the discrimination against ether lipids, mainly DAGE, in order to obtain a final product rich in DAGE and MAGE and free of glycerides. For that matter, two different types of ethanolysis reaction, namely type 1 and type 2, were compared. Ethanolysis type 1 consists of ethanol dissolved in shark liver oil and ethanolysis type 2 the opposite, shark liver oil dissolved in ethanol. Both systems provide a homogeneous single phase where the reaction takes place. In these two types of ethanolysis reaction systems, up to 5 commercial lipases were tested and compared, and the best reaction conditions for discrimination against DAGE were established. 


\section{Materials and Methods}

\subsection{Materials}

Shark liver oil was purchased from Lysi (Reykjavik, Iceland). n-hexadecane was purchased from Merck (Darmstadt, Germany). All solvents used were HPLC grade from Lab-Scan (Dublin, Ireland). Novozym 435 (immobilized lipase from Candida antarctica B) and Lypozyme RM IM from Rhizomucor miehei were purchased from Novozymes (Bagsvaerd, Denmark). Lipases SL from Burkholderia cepacea, PLG and QLC from Alcaligenes, and TL from Pseudomonas stutzeri were kindly donated by Meito Sangyo (Nagoya, Japan). Lipase G from Penicillium, Newlase F from Rhizopus niveus, and Lipase DF from Rhizopus oryzae were a gift from Amano (Nagoya, Japan). Lipase from porcine pancreas type II was purchased from Sigma Chemical Co. (St. Louis, MO).

\subsection{Enzymatic ethanolysis}

Lipase-catalyzed ethanolysis reactions of shark liver oil were performed in an orbital shaker at $240 \mathrm{rpm}$ and $35{ }^{\circ} \mathrm{C}$. Initially, a screening of different lipases was carried out to evaluate their efficiency in ethanolysis of shark liver oil. Novozym 435 from Candida antarctica, Lipases PLG and QLC from Alcaligenes, SL from Burkholderia cepacea, TL from Pseudomonas, Lypozyme RM IM from Rhizomucor miehei, lipase G from Penicillium, Newlase F from Rhizopus niveus, Lipase DF from Rhizopus oryzae and pancreatic lipase were tested. In these reactions, $2 \mathrm{~g}$ of shark liver oil were mixed with $300 \mathrm{mg}$ of absolute ethanol in $30 \mathrm{ml}$ vials. The amount of lipase used was $200 \mathrm{mg}(10 \% \mathrm{w} / \mathrm{w}$ of the oil in the reaction mixture) and $200 \mathrm{mg}$ of $\mathrm{n}$-hexadecane were added as internal standard. The efficiency of the mentioned lipases was determined by measuring the FAEE production after $10 \mathrm{~h}$ of ethanolysis reaction. 
In the next step, ethanolysis of shark liver oil was carried out only with the lipases that provided a significant extent of FAEE in the previous screening. In this step, two different oil:ethanol molar ratios were investigated:

- Ethanolysis type 1:4.34 $\mathrm{g}$ of shark liver oil were mixed with $0.66 \mathrm{~g}$ of ethanol in 60 $\mathrm{ml}$ vials.

- Ethanolysis type 2: In this case, the reactants ratio, based on the methodology reported by Irimescu et al. (Irimescu et al., 2001; Irimescu et al., 2002), consisted of $5 \mathrm{~g}$ of shark liver oil and $15 \mathrm{~g}$ of ethanol placed in $120 \mathrm{ml}$ vials.

In both types, 430-450 mg n-hexadecane was added to the reaction mixture as internal standard $(7-10 \% \mathrm{w} / \mathrm{w}$ of the oil in the reaction mixture). The amount of enzyme used was $0.5 \mathrm{~g}$ (approximately $10 \% \mathrm{w} / \mathrm{w}$ of the oil in the reaction mixture). The reactions were performed in an orbital shaker at $240 \mathrm{rpm}$ and $35^{\circ} \mathrm{C}$.

Aliquots of the reaction mixture were withdrawn periodically from the reaction mixture for the subsequent analyses by HPLC and GC. For HPLC, aliquots of $50 \mu$ in type 1 and $100 \mu \mathrm{l}$ in type 2 were taken and dissolved in $1 \mathrm{ml}$ chloroform. For GC, aliquots of $15 \mu \mathrm{l}$ in type 1 and $75 \mu$ in type 2 were taken and dissolved in $1 \mathrm{ml}$ hexane. The solutions were filtered with a nylon syringe filter $(0.45 \mu \mathrm{m})$ to completely eliminate the lipase. Then, the samples were analyzed by HPLC and GC.

\subsection{HPLC analysis}

The neutral lipids composition was determined on a Kromasil silica 60 column (5 $\mu$ m, $250 \times$ $4.6 \mathrm{~mm}$, Análisis Vínicos, Tomelloso, Spain) coupled to a HPLC Agilent 1200 Series (Avondale, PA) containing a thermostated column compartment ( $\left.35^{\circ} \mathrm{C}\right)$, a quaternary pump, an autosampler, a vacuum degasser, and evaporative light scattering detector (ELSD). Conditions of the ELSD were $2 \times 10^{5} \mathrm{~Pa}, 35^{\circ} \mathrm{C}$, and gain 3 . The flow rate was $2 \mathrm{ml} / \mathrm{min}$. A split valve was used after the column, and only $50 \%$ of the mobile phase was directed 
through the detector. The column temperature was maintained at $35^{\circ} \mathrm{C}$. The mobile phase utilized has been previously reported by Torres et al (Torres, Vázquez, Señoráns \& Reglero, 2005). This methodology permits one to analyze up to 18 different neutral lipids classes including AKG, MAGE, DAGE, sterols, tocopherols, and mono-, di-, and tri-acylglycerols. To accurately quantify minor compounds, the gain of the detector was adjusted. The products purified by semi-preparative HPLC were used to as external standards for quantification.

\subsection{GC analysis}

Fatty acid ethyl esters were analyzed by GC. $1 \mu \mathrm{L}$ of the diluted sample was injected into an Agilent (Avondale, PA) gas chromatograph (6890N Network GC System) coupled to an autosampler (Agilent 7683B). The capillary column was a $30 \mathrm{~m} \mathrm{HP-88} \mathrm{(Avondale,}$ Pennsylvania) (0.25 mm i.d.). A 20:1 split ratio was utilized. The injector and detector temperatures were 220 and $250^{\circ} \mathrm{C}$, respectively. The temperature program was as follows: starting at $100{ }^{\circ} \mathrm{C}$ and then heating to $180{ }^{\circ} \mathrm{C}$ at $20 \stackrel{\circ}{\circ} \mathrm{C} / \mathrm{min}$, followed by heating from 180 to $220^{\circ} \mathrm{C}$ at $15^{\circ} \mathrm{C} / \mathrm{min}$. The final temperature $\left(220^{\circ} \mathrm{C}\right)$ was held for $10 \mathrm{~min}$. Identification of the various fatty acids was based on the retention times and relative area percentages of a PUFA No. 3 standard (-7085) obtained from Supelco. These retention times and areas percentages were compared with those obtained when shark liver oil was properly chemically transesterified in the presence of sodium ethoxide. Quantification was effected via an internal standard of $n$-hexadecane.

Shark liver oil was comprised of 2\% C14:0, 17\% C16:0, 6\% C16:2, 3\% C18:0, 34\% C18:1, 10\% C20:1, 2\% C20:5, 10\% C22:1, 7\% C22:6 and 4\% C24:1. Other minor fatty acids (<2\%) contributed to $100 \%$ of total composition.

\subsection{Purification of standards by semi-preparative HPLC}


Since there were not commercially available standards of the different compounds involved in shark liver oil ethanolysis reactions, each of these lipid classes was purified by semipreparative HPLC for an accurate identification and quantification via HPLC coupled to ELSD detector. Hence, TAG, diacylglycerols, monoacylglycerols, DAGE, MAGE, and AKG were isolated at $\mathrm{mg}$ scale. Purification of the mentioned compounds was perfomed by using a semi-preparative Kromasil silica column $(5 \mu \mathrm{m}, 250 \times 10$ mm, Análisis Vínicos, Tomelloso, Spain) coupled to the HPLC system. Some characteristics of the analytical HPLC method were adapted to the semi-preparative process: Concentration of samples $(50 \mathrm{mg} / \mathrm{ml})$, injection volume $(500 \mu \mathrm{l})$, and flow rate $(5.8 \mathrm{ml} / \mathrm{min})$.

TAG and DAGE were obtained by injecting the original shark liver oil. MAGE and diacylglycerols were purified by injecting the product mixture attained after $7 \mathrm{~h}$ of shark liver oil ethanolysis catalyzed by Novozym 435, which provided high relative area of the different intermediate compounds from ethanolysis reaction in the chromatogram. To collect the fractions, an Agilent 1200 Series Analytical and Preparative Scale Fraction Collectors (Avondale, PA) coupled to HPLC was used.

\subsection{Water content analysis of lipases}

Karl Fischer automatic titrator 870 Titrino Plus (Metrohm, Herisau, Switzerland) with hydranal composite 5 (Sigma Chemical Co., St. Louis, MO) as titration reagent, was used to determine the water content of the immobilized lipases.

\subsection{Enzymatic residual activity analysis}

The residual activity of catalysts was tested by performing an alcoholisis reaction with each of the biocatalyst recovered from shark liver ethanolysis product mixture. Hence, $434 \mathrm{mg}$ of olive oil was mixed with $66 \mathrm{mg}$ of absolute ethanol and $40 \mathrm{mg}$ of $\mathrm{n}$-hexadecane as internal standard. The reaction was performed in $10 \mathrm{ml}$ vials placed in an orbital shaker at $240 \mathrm{rpm}$ 
and $35{ }^{\circ} \mathrm{C}$. After 30 minutes, the reaction product was analyzed by $\mathrm{GC}$ as previously described to determine the amount of ethyl esters obtained. The activity units were defined as $\mu$ mols of ethyl ester produced / $\mathrm{min} / \mathrm{g}$ lipase.

\section{Results and discussion}

\subsection{Lipase screening}

In biocatalysis, one of the first parameter to look at is the reaction conversion. In lipasecatalyzed ethanolysis reactions this variable can be measured by the percentage of disappearance of the reactant or by the percentage of apparition of the products. In order to establish which lipase is more efficient in shark liver oil ethanolysis, regardless of the acyl donor preferentially utilized, apparition of FAEE was first considered (Martín et al.).

It can be observed that up 10 different commercial lipases were compared as biocatalysts in shark liver oil ethanolysis. The highest conversion was attained with lipase TL. After this study, the 5 biocatalysts that produced higher percentage in FAEE were selected for further experiments.

\subsection{Ethanolysis type 1}

Ethanolysis reactions can be carried out in homogeneous system, under two markedly different reaction conditions: type 1) in which ethanol is dissolved in oil, and type 2) in which oil is dissolved in ethanol. In order to evaluate the activity and selectivity of different lipases in ethanolysis reactions both strategies should be compared. In shark liver oil, there are two lipid classes that can undergo ethanolysis, namely DAGE and TAG. The rate of disappearance of DAGE and TAG in the presence of the five commercial lipases that exhibited higher rate of ethanolysis reaction is depicted in Figure 2. 
It should be noted that all lipases under study except lipase SL showed lower rate of ethanolysis towards DAGE than that towards TAG. Lipase SL showed two clearly differentiated stages in the time course of the ethanolysis reaction: a first stage with almost negligible reaction conversion and a second stage, after 10 hours of ethanolysis reaction, with markedly faster reaction rate towards TAG than that towards DAGE.

In addition, lipase TL was the fastest lipase of all tested. Total disappearance of TAG was attained in less than one hour of ethanolysis reaction in the presence of this lipase. Besides, the reaction conversion of DAGE and TAG for the different lipases assayed should be also considered. The reaction conversion for TAG was higher than $90 \%$ for all lipases investigated. On the contrary the reaction conversion of DAGE was $73 \%, 82 \%, 91 \%, 66 \%$, and $60 \%$ for Novozym 435, SL, TL, QLC, and PLG, respectively.

Another important aspect in ethanolysis reaction is chemo-selectivity. In other words, it should be evaluated if the different lipases under study act preferentially on triradylglycerols, on diradylglycerols, on monoradylglycerol or indiscriminately among them. In this sense, two major ethanolysis product to look at, are 1,2-DAG and 1-MAGE. If sufficient ethanol is present in the mixture these two products can undergo a new ethanolysis reaction to produce 2-MAG and AKG. However under the experimental conditions assayed the main products of ethanolysis were 1,2-DAG and 1-MAGE. The apparition of these two compounds for the 5 lipases investigated is depicted in Figure 3.

Analyzing 1,2-DAG and MAGE produced by the 5 lipases under study there are several differentiated trends. Hence, lipases QLC and PLG showed similar pattern for 1,2DAG and MAGE. These two species reach a plateau after ca. 8 hours of ethanolysis reaction and it is kept almost constant along the course of the reaction. On the contrary 1,2-DAG never exceeded $5 \%(\mathrm{w} / \mathrm{w})$ in the reaction mixture in the presence of Novozym 435 and $\mathrm{SL}$ lipases. This result can be attributed to a rapid ethanolysis of 1,2 DAG as soon as they are formed in the reaction mixture. Surprisingly, MAG are not accumulated in the product 
mixture and they never exceeded $5 \%(\mathrm{w} / \mathrm{w})$ in the mixture. This result could also indicate that these lipases act preferentially on DAG and MAG and they are transformed into FAEE and glycerol as soon as they are produced in the ethanolysis reaction. One important difference between these two lipases is that after 24 hours of ethanolysis reaction percentage of MAGE start decreasing for lipase $\mathrm{SL}$ and was constant and stable for Novozym 435. Finally in the presence of lipase TL both 1,2-DAG and MAGE showed a rapid increase and posterior decrease in less than 8 hours. In conclusion, maximum discrimination against MAGE was observed for ethanolysis reaction type 1 catalyzed by Novozym 435, that kept untouched MAGE even after 48 hours of reaction.

\subsection{Ethanolysis type 2}

Previously described ethanolysis reactions, were carried out by using conditions in which ethanol is the minor compound dissolved in the oil that act as the major compound in the reaction mixture. On the contrary, the results obtained utilizing ethanol as the most abundant compound in the reaction mixture, are shown in Figure 4.

Surprisingly, at these reaction conditions lower reaction rates were observed for all lipases except Novozym 435. This result could indicate a more pronounced deleterious effect of ethanol at this concentration on lipase activity and stability. These lower reaction rates could be also attributed to a lower enzyme percentage $(w / v)$ in the reaction mixtures. The total reaction conversion for TAG was attained for all lipases investigated except for lipase SL. Regarding DAGE, reaction conversion was ca. $100 \%, 25 \%, 85 \%, 80 \%$, and $75 \%$ for Novozym 435, SL, TL, QLC, and PLG, respectively. These results indicate lower discrimination against DAGE when excess of ethanol is utilized. Besides, utilization of ethanol as the major compound in the reaction mixture reduces significantly the grams of product per liter of reaction mixture and per time unit (volumetric productivity). However, chemo-selectivity of the ethanolysis reaction should be also taken into consideration. For 
that reason formation of diradylglycerols such as 1,2-DAG and MAGE (Figure 5) should be also monitored before taking any decision regarding which procedure of ethanolysis is the most convenient.

Under these reaction conditions, Novozym 435 produced more than $10 \%(\mathrm{w} / \mathrm{w})$ of $1,2-D A G$ in contrast to less of $5 \%$ of this chemical specie produced when ethanol was present as a minor compound. However, similarly to the results showed in Figure 2 after 24 hours of ethanolysis in the presence of Novozym 435, almost total disappearance of 1,2DAG was observed. Lipases QLC and PLG exhibited similar behavior to that observed when ethanol was present as a minor compound except that in the presence of PLG, MAGE start decreasing after 24 hours reaching a final value of ca. $10 \%(\mathrm{w} / \mathrm{w})$.

Regarding monoradylglycerols (Figure 6) only monoacylglycerols were detected when ethanol was utilized as the major compound in the reaction mixture. In addition, in the presence of lipase TL significant amount of AKG were also observed. Lipase SL did not produced significant amounts of monoradylglycerols under these reaction conditions.

It should be noted that these reaction conditions are more suitable for production of monoacylglycerols as were previously described by Irimescu et al (Irimescu et al., 2001). However in ethanolysis of shark liver oil monoradylglycerols attained from MAGE were not significantly produced except in the presence of lipase TL. For this reason chemo-selectivity towards both types of monoradylglycerols could not be properly studied. This result also indicates that most lipases utilized did not catalyze ethanolysis of MAGE that is accumulated in the reaction mixture. Similar rate of apparition of both monoradylglycerols was observed in ethanolysis catalyzed by lipase TL which indicates no clear discrimination against MAGE.

\subsection{Lipase stability}

Another important aspect in lipase-catalyzed ethanolysis reactions is the stability of the biocatalyst. In order to evaluate the residual activity of each lipase after ethanolysis, each 
enzyme was recovered, washed and dried, and subsequently utilized in an ethanolysis test reaction in the presence of olive oil. The activity units of each fresh lipase and that after the two ethanolysis reaction studied are shown in Table 2.

The most active biocatalyst assayed was Novozym 435. After ethanolysis reaction the residual activity was ca. $97 \%$ in both types of ethanolysis studied. The rest of biocatalysts assayed showed very low residual activity after ethanolysis type 2 (ca. 10\%) except lipase SL that was completely inactivated after both types of ethanolysis. Ethanolysis type 1 preserves almost intact residual activity of most lipases investigated which is an advantage of this procedure in comparison with ethanolysis type 2 taking into consideration that biocatalyst is the most expensive ingredient of the ethanolysis reaction mixture.

\subsection{Water content and glycerol content}

Another important aspect is water content of the biocatalyst and glycerol content in the course of the ethanolysis reaction. The initial water content of all lipases assayed is shown in Table 3. It should be noted that immobilized enzymes (Novozym 435, QLC, and PLG) contains lower amount of water than non immobilized lipases (SL and TL).

Regarding glycerol content it has become common knowledge that it has a negative effect on lipase activity and stability likely by being adsorbed onto the support of the immobilized lipases and reducing the diffusion of the hydrophobic substrate to the active site of the lipase (Xu et al., 2011). For this reason the glycerol accumulation as a function of the reaction time was determined. Each data point on the glycerol production curve was calculated from the compositions of FFA, FAEE, TAG, DAG and MAG at the corresponding time point, as measured by HPLC. The glycerol composition for ethanolysis types 1 and 2 are depicted in Figure 7. It can be observed that higher percentages of glycerol were produced in ethanolysis type 1 than in ethanolysis type 2. In addition those lipases that consumed more than $90 \%$ of TAG and 1,2-DAG (Novozym 435, SL, and TL) produced ca. 
$5 \%$ of glycerol in the mixture in ethanolysis type 1 . This result also indicates that MAG is not accumulated in the product mixture and it is rapidly transformed in their corresponding FAEE and glycerol. On the contrary ethanolysis type 2 only produced $4 \%(w / w)$ of glycerol in the presence of Novozym 435 after 48 hours of reaction. This result indicate that total conversion of TAG, DAG and MAG is not achieved under these conditions and as it was previously mentioned in ethanolysis type 2 this lipase preferentially accumulates MAG in the first 10 hours of ethanolysis reaction.

\section{Conclusions}

All lipases tested showed discrimination against DAGE in lipase-catalyzed ethanolysis reactions of shark liver oils. Based on DAGE conversion it can be also concluded that lower discrimination against DAGE was observed for ethanolysis type 2. Regarding diradylglycerols lipases PLG and QLC accumulates both 1,2-DAG and MAGE in the reaction mixtures and on the contrary, Novozym 435 discriminates against MAGE. Regarding monoradylglycerols they were only detected in significant amounts in ethanolysis type 2 . Similar rate of apparition was observed for monoacylglycerols and for AKG in the presence of lipase TL. Ethanolysis type 2 is more adequate for production of monoacylglycerols although it produces significant inactivation of most lipases except Novozym 435 . On the contrary, inactivation of lipase was almost negligible for ethanolysis type 1 except for lipase SL that lost completely the catalytic activity in both types of ethanolysis assayed. In conclusion, it can be summarized that the best reaction conditions for achieving discrimination against ether lipids and higher lipase stability are ethanolysis type 1 in the presence of Novozym 435.

\section{Acknowledgements}


This work was supported by Comunidad Autónoma de Madrid (ALIBIRD, project number S2009/AGR-1469) and Consolider-Ingenio FUN-C-FOOD (CSD2007- 00063). A predoctoral contract for Óscar Fernández (Universidad Autonoma de Madrid) is also acknowledged.

\section{References}

Andreesen, R. (1988). Ether lipids in the therapy of cancer. Prog Biochem Pharmacol, 22, 118-131.

Böer, M., Gannefors, C., Kattner, G., Graeve, M., Hop, H., \& Falk-Petersen, S. (2005). The arctic pteropod Clione limacina: seasonal lipid dynamics and life-strategy. Mar Biol, 147(3), 707-717.

Breivik, H., Haraldsson, G., \& Kristinsson, B. (1997). Preparation of highly purified concentrates of eicosapentaenoic acid and docosahexaenoic acid. J Am Oil Chem Soc, 74(11), 1425-1429.

Diomede, L., Colotta, F., Piovani, B., Re, F., Modest, E. J., \& Salmona, M. (1993). Induction of apoptosis in human leukemic cells by the ether lipid 1-octadecyl-2-methyl-RAC-glycero3-phosphocholine. A possible basis for its selective action. Int J Cancer, 53(1), 124-130.

Halldorsson, A., Kristinsson, B., \& Haraldsson, G. G. (2004). Lipase selectivity toward fatty acids commonly found in fish oil. Eur J Lipid Sci Techol, 106(2), 79-87.

Haraldsson, G., \& Kristinsson, B. (1998). Separation of eicosapentaenoic acid and docosahexaenoic acid in fish oil by kinetic resolution using lipase. J Am Oil Chem Soc, 75(11), 1551-1556.

Haraldsson, G., Kristinsson, B., Sigurdardottir, R., Gudmundsson, G., \& Breivik, H. (1997). The preparation of concentrates of eicosapentaenoic acid and docosahexaenoic acid by 
lipase-catalyzed transesterification of fish oil with ethanol. J Am Oil Chem Soc, 74(11), 14191424.

Hayashi, K., \& Kishimura, H. (2002). Amount and composition of diacyl glyceryl ethers in various tissue lipids of the deep-sea squid Berryteuthis magister. J Oleo Sci, 51(8), 523-530.

Imbs, A., Demina, O., \& Demidkova, D. (2006). Lipid class and fatty acid composition of the boreal soft coral soft coral Gersemia rubiformis. Lipids, 41(7), 721-725.

Irimescu, R., Furihata, K., Hata, K., Iwasaki, Y., \& Yamane, T. (2001). Utilization of reaction medium-dependent regiospecificity of Candida antarctica lipase (Novozym 435) for the synthesis of 1,3-dicapryloyl-2-docosahexaenoyl (or eicosapentaenoyl) glycerol. J Am Oil Chem Soc, 78(3), 285-290.

Irimescu, R., Iwasaki, Y., \& Hou, C. (2002). Study of TAG ethanolysis to 2-MAG by immobilized Candida antarctica lipase and synthesis of symmetrically structured TAG. J Am Oil Chem Soc, 79(9), 879-883.

Jensen, R., DeJong, F., \& Clark, R. (1983). Determination of lipase specificity. Lipids, 18(3), 239-252.

Magnusson, C. D., \& Haraldsson, G. G. (2011). Ether lipids. Chem Phys Lipids, 164(5), 315340.

Martín, D., Morán-Valero, M., Señoráns, F., Reglero, G., \& Torres, C. (2011). In vitro intestinal bioaccessibility of alkylglycerols versus triacylglycerols as vehicles of butyric acid. Lipids, 46(3), 277-285.

Meimetis, L. G., Williams, D. E., Mawji, N. R., Banuelos, C. A., Lal, A. A., Park, J. J., Tien, A. H., Fernandez, J. G., de Voogd, N. J., Sadar, M. D., \& Andersen, R. J. (2011). Niphatenones, glycerol ethers from the sponge Niphates digitalis block androgen receptor transcriptional activity in prostate cancer cells: structure elucidation, synthesis, and biological activity. J Med Chem, 55(1), 503-514. 
Palmblad, J., Samuelsson, J., \& Brohult, J. (1990). Interactions between alkylglycerols and human neutrophil granulocytes. Scand J Clin Lab Invest, 50(4), 363-370.

Piyatheerawong, W., Iwasaki, Y., Xu, X., \& Yamane, T. (2004). Dependency of water concentration on ethanolysis of trioleoylglycerol by lipases. $J$ Mol Catal B-Enzym, 28(1), 1924.

Senanayake, S. P. J. N., \& Shahidi, F. (2000). Concentration of docosahexaenoic acid (DHA) from algal oil via urea complexation. J Food Lipids, 7(1), 51-61.

Shimada, Y., Sugihara, A., \& Tominaga, Y. (2001). Enzymatic purification of polyunsaturated fatty acids. J Biosci Bioeng, 91(6), 529-538.

Song, X., Qi, X., Hao, B., \& Qu, Y. (2008). Studies of substrate specificities of lipases from different sources. Eur J Lipid Sci Technol, 110(12), 1095-1101.

Tenllado, D., Reglero, G., \& Torres, C. F. A combined procedure of supercritical fluid extraction and molecular distillation for the purification of alkylglycerols from shark liver oil. Sep Purif Technol, 83(0), 74-81.

Torres, C. F., Vázquez, L., Señoráns, F. J., \& Reglero, G. (2009). Enzymatic synthesis of short-chain diacylated alkylglycerols: A kinetic study. Process Biochem, 44(9), 1025-1031.

Torres, C. F., Vazquez, L., Senorans, F. J., \& Reglero, G. (2007). An efficient methodology for the preparation of alkoxyglycerols rich in conjugated linoleic acid and eicosapentaenoic acid. J Am Oil Chem Soc, 84(5), 443-448.

Torres, C. F., Vázquez, L., Señoráns, F. J., \& Reglero, G. (2005). Study of the analysis of alkoxyglycerols and other non-polar lipids by liquid chromatography coupled with evaporative light scattering detector. J Chromatogr A, 1078(1-2), 28-34.

Vázquez, L., \& Akoh, C. C. (2010). Fractionation of short and medium chain fatty acid ethyl esters from a blend of oils via ethanolysis and short-path distillation. $J$ Am Oil Chem Soc, 87(8), 917-928. 
Vázquez, L., \& Akoh, C. C. (2011). Concentration of stearidonic acid in free fatty acid and fatty acid ethyl ester forms from modified soybean oil by winterization. $J$ Am Oil Chem Soc, $88(11), 1775-1785$.

Vázquez, L., Fornari, T., Señoráns, F. J., Reglero, G., \& Torres, C. F. (2008). Supercritical carbon dioxide fractionation of nonesterified alkoxyglycerols obtained from shark liver oil. $J$ Agric Food Chem, 56(3), 1078-1083.

Watanabe, Y., Shimada, Y., Sugihara, A., \& Tominaga, Y. (1999). Stepwise ethanolysis of tuna oil using immobilized Candida antarctica lipase. J Biosci Bioeng, 88(6), 622-626.

Xu, Y., Nordblad, M., Nielsen, P. M., Brask, J., \& Woodley, J. M. (2011). In situ visualization and effect of glycerol in lipase-catalyzed ethanolysis of rapeseed oil. J Mol Catal B-Enzym, 72(3-4), 213-219.

\section{Figure Captions}

Figure 1. Chemical structure of the main subclasses of triradyl-, diradyl- and monoradylglycerols. Positional isomers are not included in the figure.

Figure 2. Time course of the disappearance of DAGE and TAG in the presence of the five commercial lipases investigated under ethanolysis type 1. 
Figure 3. Time course of the apparition of 1,2-DAG and 1-MAGE for the 5 lipases investigated under ethanolysis type 1.

Figure 4. Time course of the disappearance of DAGE and TAG in the presence of the five commercial lipases under ethanolysis type 2.

Figure 5. Time course of the apparition of 1,2-DAG and 1-MAGE for the 5 lipases investigated under ethanolysis type 2 .

Figure 6. Time course of the formation of monoradylglycerols in the presence of different commercial lipases.

Figure 7. Glycerol production estimated as function of time in the presence of 5 different commercial lipases.

Table 1. Percentage of FAEE produced after 10 hours of ethanolysis reaction for the different lipases investigated

\begin{tabular}{|l|c|}
\hline Biocatalyst & \% FAEE (w/w) \\
\hline Lypozyme RM IM (Rhizomucor miehei) & 4.1 \\
\hline Lipase QLC (Alcaligenes) & 28.2 \\
\hline Lipase G (Penicillium) & 0.9 \\
\hline Newlase F (Rhizopus niveus) & 0.8 \\
\hline Lipase DF (Rhizopus oryzae) & 1.2 \\
\hline Lipase SL (Burkholderia cepacea) & 6.7 \\
\hline Lipase TL (Pseudomonas) & 48.4 \\
\hline
\end{tabular}




\begin{tabular}{|l|c|} 
Lipase PLG (Alcaligenes) & 29.6 \\
\hline Novozym 435 (Candida antarctica) & 46.2 \\
\hline Porcine pancreatic lipase & 1.1 \\
\hline
\end{tabular}

Table 2. Residual activity of each lipase before and after ethanolysis reaction

\begin{tabular}{|l|c|c|c|}
\cline { 2 - 4 } \multicolumn{1}{c|}{} & \multicolumn{3}{c|}{ Activity Units } \\
\hline Biocatalyst & Fresh & Ethanolysis type 1 & Ethanolysis type 2 \\
\hline Novozym 435 & 28.10 & 28.12 & 28.16 \\
\hline SL & 25.1 & 8.0 & 3.2 \\
\hline TL & 595 & 375.3 & 377.2 \\
\hline QLC & 105.2 & 61.4 & 10.0 \\
\hline PLG & 154.8 & 153.1 & 14.0 \\
\hline
\end{tabular}


Table 3. Water content of the biocatalysts utilized

\begin{tabular}{|l|c|}
\hline Biocatalyst & \% water content (w/w) \\
\hline Novozym435 & 0.9 \\
\hline SL & 11.7 \\
\hline TL & 11.3 \\
\hline QLC & 1.2 \\
\hline PLG & 0.9 \\
\hline
\end{tabular}

